Tropical Journal of Pharmaceutical Research August 2012; 11 (4): 657-664

(C) Pharmacotherapy Group, Faculty of Pharmacy, University of Benin Benin City, 300001 Nigeria.

All rights reserved.

Available online at http://www.tjpr.org http://dx.doi.org/10.4314/tjpr.v11i4.18

\title{
Adherence to Surgical Site Infection Guidelines in Cardiac Surgery in a Tertiary Hospital in Dubai, United Arab Emirates
}

\author{
Eman Abu-Gharbieh ${ }^{1^{*}}$ and Sahar Fahmy ${ }^{2}$ \\ ${ }^{1}$ Dubai Pharmacy College, Dubai, United Arab Emirates, ${ }^{2}$ Helwan University, Helwan, Egypt.
}

\begin{abstract}
Purpose: To assess the appropriateness and compliance of antibiotic prophylaxis practices in cardiac surgery in a tertiary hospital in United Arab Emirates (UAE) using three international guidelines.

Methods: A retrospective study was performed by reviewing patients' files admitted for cardiac surgery between January 2008 and February 2010. The study evaluated the adherence of health care professionals to three international guidelines with regard to antibiotic prophylaxis. The guidelines were National Surgical Infection Prevention Project (NSIPP), Society of Thoracic Surgeons (STS) and American College of Cardiology/American Heart Association (ACC/AHA). Patients' records were reviewed for antibiotics used for prophylaxis, frequency of administration, timing and number of doses.

Results: A total of 92 patients were included in the study. Based on the international guidelines, only 89.1 and $79.3 \%$ of the patients received the recommended pre- and post-operative antibiotics, respectively. On the other hand, $93.5 \%$ of the patients received the right antibiotic dose while the total duration of all antimicrobial agents used for prophylaxis was concordant with the guidelines (48 h) in only $67.4 \%$ of the patients.

Conclusion: Adherence to international antimicrobial prophylaxis guidelines for cardiac surgery was found to be suboptimal in the study hospital in Dubai. Various interventions are needed via developing local evidence-based protocols in collaboration with surgeons, and also to strengthen regulations for ensuring adherence to these guidelines.
\end{abstract}

Keywords: Antimicrobial prophylaxis, International guidelines, Cardiac surgery 


\section{INTRODUCTION}

Surgical site infection (SSI) represents one of the most common nosocomial infections; its incidence rate is estimated to be in the range of $2-5 \%$ in patients undergoing clean extraabdominal operations and up to $20 \%$ in patients undergoing intra-abdominal operations [1]. Although cardiac surgery is generally considered a clean procedure, several studies have shown that significant morbidity and mortality are associated with major cardiovascular surgery [2]. Antibiotic prophylaxis has been demonstrated to lower the incidence of SSI [3].

The vast body of literature addressing the prevention of the incidence of SSls have been summarized and published in several international guidelines to properly direct physicians and other health care providers in their clinical decision making by describing the generally accepted approaches for the diagnosis, management or prevention of certain disease conditions [4]. Among these are the American College of Cardiology/American Heart Association (ACC/AHA) [5], the National Surgical Infection Prevention Project (NSIPP) [6] and the Society of Thoracic Surgeons (STS) [3,4]. The three international guidelines are well accepted standards published and can easily be accessed for guidance in antimicrobial prophylaxis in cardiac surgery. There are no major differences between the selected international guidelines.

The recommendations made by these guidelines can be summarized as follows: a) antimicrobial prophylaxis should be given to all patient undergoing cardiac surgeries, b) the antibiotics of choice are first or second generation cephalosporin (cefazolin or cefuroxime), c) vancomycin should be reserved for patients with known cases of methicillin-resistant Staphylococcus aureus (MRSA) or for patients with allergy to betalactams, d) the duration of antimicrobial prophylaxis should be no longer than $48 \mathrm{~h}$ with the first dose given to the patient within
$60 \mathrm{~min}$ prior to skin incision. Therefore, the timing for antimicrobial prophylaxis is considered optimal if administered within 30 to 60 minutes before incision.

In the United Arab Emirates (UAE), there is no national drug policy in place and antimicrobial prophylaxis in cardiac surgery and hence there is no standard protocol that cuts across all health facilities in UAE. Several non-unified local protocols exist in different health care facilities regarding antimicrobial prophylaxis in cardiac surgery. Studies on current practice of antimicrobial prophylaxis in cardiac surgery appear to be lacking in UAE and there is a need to generate baseline data on the pattern of prophylactic antibiotic use in cardiac surgery. The purpose of this study was to assess the appropriateness and compliance of antibiotic prophylaxis practices in cardiac surgery to three international guidelines (NSIPP, STS and $A C C / A H A$ ) in a tertiary hospital in UAE.

\section{EXPERIMENTAL}

\section{Setting and study design}

This study was conducted in a tertiary 600bed hospital providing general medical and surgical services. The hospital is located in Dubai which is one of the largest cities (by area and population) in the United Arab Emirates (UAE).

Following approval by the Research Committee in Dubai Pharmacy College, the case files of patients admitted for cardiac surgery during the period of January 2008 and February 2010 were reviewed retrospectively. The files included in the study were those of patients who had cardiac surgery. Patients who were diagnosed with human immunodeficiency virus (HIV) infection, tuberculosis, or cystic fibrosis were excluded. Patient records documenting suspected infections other than SSI which required antibiotic administration were also excluded from the study. 


\section{Data collection}

Data were collected from patients' records, medication sheets, and prescriptions using a clinical data collection form. The developed form was tested prior to the study on a small pilot sample $(n=8)$ and subsequently modified to ensure that the data are valid. The data collection form had two sections. Section I included 1) Patient demographic information, i.e., patient's file number, gender, age, weight, date of admission and date of discharge; and 2) Medical information, i.e., diagnosis, type of surgery, past medical history, drug history, drug allergy, antibiotic use in the last 2 weeks and previous hospitalization in the last 2 years. Section II included 1) antibiotic use or nonuse and type of prophylactic antibiotic(s) used during surgery incorporating scientific name, dose, frequency, timing of doses, route of administration, duration of use, and number of doses of all antibiotics used (pre-, intra-, and post-operatively) as surgical prophylactic antibiotics; and 2) duration of surgery in minutes.

A total of 108 patient records of those who had cardiac surgery were identified during the study period. Five records were excluded from the study; three of these records were for patients with tuberculosis, one with pneumonia and one with suspected infection that required antibiotic therapy. Additional 11 patient records could not be evaluated due to incomplete or missing information. In all, 92 patient records were thus eligible for the study.

\section{Data analysis}

Data were coded, entered into a spreadsheet and analyzed using SPSS ${ }^{\circledR}$ version 16 (SPSS Inc., Chicago, IL). Descriptive statistics were carried out for all assessed parameters and, where appropriate, the results were expressed as mean \pm standard deviation.

\section{RESULTS}

Of the 92 patients included in the study, 73 were males while 19 were females. The average age of the patients was $48.7 \pm 19.3$ years (median $=51$ years) and the mean weight was $15.9 \pm 22.2 \mathrm{~kg}$. About $40 \%$ of the patients had normal body weight (BMI, 18.5 $24.9 \mathrm{~kg} / \mathrm{m}^{2}$ ), $33.7 \%$ were overweight, $16.3 \%$ obese and $9.8 \%$ underweight. About $32.0 \%$ of the patients were smokers and $5.4 \%$ quit smoking a year earlier. A majority of the patients $(84.8 \%)$ currently drink coffee while $9.8 \%$ take alcoholic beverages. About half $(53.3 \%)$ of the patients were diagnosed with three or more medical conditions. The majority of patients $(78.3 \%)$ were admitted for coronary artery bypass with a mean surgery time of $4.6 \pm 1.9 \mathrm{~h}$. Table 1 summarizes the data.

\section{Indication}

Antibiotic prophylaxis was received by 90 patients $(97.8 \%)$ while post-operative doses were given to 87 patients $(94.6 \%)$ who had cardiac surgery.

\section{Antibiotic treatment}

\section{Pre-operative therapy}

Antibiotic choice pre-operation was in accordance to guidelines in $89.1 \%$ of patients who received cefuroxime while the antibiotic choice in 10 patients (10.9\%) did not adhere to guidelines due to (a) three patients were given cefuroxime even when they had documented beta-lactam allergy, (b) unexplained administration of cefuroxime and metronidazole combination in one patient, (c) unexplained use of piperacillin sodium and tazobactam odium as pre-operative prophylactic antimicrobial agent in four patients, and (d) no administration of antimicrobial prophylactic dose in two patients due to urgency of the cardiac surgery The data obtained are presented in Table 2. 
Table 1: Characteristics and types of cardiac surgeries for enrolled patients

\begin{tabular}{ll}
\hline Characteristic & $\mathbf{N}(\%)$ \\
\hline Mean age (years) & $48.7 \pm 19.3$ \\
Gender & \\
Male & $73(67.16)$ \\
Female & $19(17.48)$ \\
Patient Average weight $(\mathrm{kg})$ & $66.0 \pm 22.0$ \\
Underweight (BMl $\left.=18.5 \mathrm{~kg} / \mathrm{m}^{2}\right)$ & $9(9.8)$ \\
Normal weight $\left(\mathrm{BMl}=18.5-24.9 \mathrm{~kg} / \mathrm{m}^{2}\right)$ & $37(40.2)$ \\
Overweight $\left(\mathrm{BMl}=25-29.9 \mathrm{~kg} / \mathrm{m}^{2}\right)$ & $31(33.7)$ \\
Obese (BMI=. 30kg/m $\left.{ }^{2}\right)$ & $15(16.3)$ \\
Mean length of hospital stay before surgery (days) & $5.5 \pm 3.3$ \\
Mean total length of hospital stay (days) & $18.2 \pm 10.6$ \\
Hospitalization in the past 2 years & $46(50)$ \\
Diabetes mellitus & $46(50)$ \\
Current smoker & $29(31.5)$ \\
Currently drinks coffee & $78(84.8)$ \\
Drinks alcohol & $9(9.8)$ \\
Three medical diagnoses or more & $49(53.3)$ \\
Type of cardiac surgery & \\
Congenital heart surgery & \\
Valve replacement surgery & $12(13)$ \\
Coronary artery bypass surgery & $6(6.5)$ \\
Other surgeries & $72(78.3)$ \\
Mean duration of surgery (hours) & $2(2.2)$ \\
\hline
\end{tabular}

Table 2: Antimicrobials utilized pre- and post-surgical operations.

\begin{tabular}{ll}
\hline Treatment & $\mathbf{N}(\%)$ \\
\hline Pre-operative & \\
Cefuroxime & $82(89.1)$ \\
Cefuroxime although documented B-lactam allergy is present & $3(3.3)$ \\
Cefuroxime and metronidazole combination & $1(1.09)$ \\
Other therapeutic category (Piperacillin sodium and tazobactam sodium combination) & $4(4.35)$ \\
No treatment due to urgency & $2(2.17)$ \\
Subtotal & $\mathbf{9 2 ( 1 0 0 )}$ \\
\hline Post-operative & \\
Cefuroxime & $73(79.3)$ \\
Levofloxacin+ flucloxacillin & $1(1.1)$ \\
Cefuroxime + Levofloxacin & $1(1.1)$ \\
Cefuroxime + Ciprofloxacin & $1(1.1)$ \\
Cefuroxime + Doxycycline & $1(1.1)$ \\
Cefuroxime + (Piperacillin sodium and tazobactam Sodium combination) & $3(3.26)$ \\
(Piperacillin sodium and tazobactam sodium combination) & $2(2.17)$ \\
(Piperacillin sodium and tazobactam sodium combination)+Levofloxacin & $1(1.10)$ \\
(Piperacillin sodium and tazobactam sodium combination)+Levofloxacin+ Cefuroxime & $4(4.35)$ \\
None & $5(5.43)$ \\
Subtotal & $\mathbf{9 2 ( 1 0 0 )}$ \\
\hline
\end{tabular}




\section{Post-operative therapy}

After surgery, almost $79.3 \%$ of the patients were given the correct antibiotic treatment (cefuroxime) with only $15.2 \%$ of patients switched from appropriate antibiotic to an inappropriate agent as shown in Table 2.

\section{Dose}

The dose given was in accordance with guidelines in 86 patients (93.5\%), as shown in Table 3. In the remaining six patients $(6.5 \%)$, the dose was not in agreement with the guidelines' recommendations on the calculated dose per body weight for four patients. Two patients did not take any antibiotic due to urgency in performing the operations.

\section{Total duration of antimicrobial prophylaxis}

In 62 out of 92 patients $(67.4 \%)$, the total duration of use of all antimicrobial agents for prophylaxis was concordant with the guidelines (48 h) while in 30 patients (32.6\%) the duration was longer than that recommended.

\section{Dosing interval}

Intra-operative dose was given to 17 patients out of the 92 patients (18.5\%). The duration of surgical procedure was equal $\leq 3 \mathrm{~h}$ in 12 patients out of the 17 patients; no additional dose of the prophylactic agent was required intra-operatively as per the guidelines. On the other hand, no intra-operative dose was administered to 75 patients $(81.5 \%) ; 74$ patients were subjected to surgical procedure longer than $3 \mathrm{~h}$ (average duration $=7 \mathrm{~h}$ ). The data are presented in Table 3.

\section{Timing of doses given pre-operatively}

For doses given pre-operatively (Table 3), timing was in accordance to the guidelines (60 min before incision) in only $30.4 \%$ ( $n=$ 28 ) of the patients while it was given $<60 \mathrm{~min}$ in $58.7 \%(n=54)$ and $>60 \mathrm{~min}$ in $8.7 \%(\mathrm{n}=$ $8)$. The remaining two patients $(2.2 \%)$ did not

Table 3: Adherence to the guidelines on dose, duration of antimicrobial use, dosing interval and dosing time

\begin{tabular}{lr}
\hline Parameter & $\mathbf{N}(\%)$ \\
\hline Dose & \\
$\quad$ In accordance with guidelines & \\
$\quad$ Dose n Not in accordance with guidelines & $6(6.5)$ \\
Duration of antimicrobials use & \\
$\quad$ In accordance with guidelines & $62(67.4)$ \\
$\quad$ Not in accordance with guidelines & $30(32.6)$ \\
Dosing intervals & \\
$\quad$ Intra-operative dose & $5(5.4)$ \\
In accordance with guidelines & $12(13)$ \\
$\quad$ Not in accordance with guidelines & \\
$\quad$ Not intra-operative dose & $1(1.2)$ \\
In accordance with guidelines & $74(80.4)$ \\
Not in accordance with guidelines & \\
Time of dosing & $28(30.4)$ \\
In accordance to guidelines (30-60 min) & $54(58.7)$ \\
Not in accordance with guidelines (<30 min) & $8(8.7)$ \\
Not in accordance with guidelines $(>60$ min) & $2(2.2)$ \\
\hline
\end{tabular}


take any prophylactic dose due to the emergency nature of the surgical procedure.

\section{DISCUSSION}

The principal finding of our study was that adherence to guidelines for antibiotic prophylaxis in cardiac surgery was not achieved $100 \%$ for all parameters in any of the 92 cardiac surgeries assessed in the study. However, these results are not surprising and are consistent with similar results obtained from studies done in some Middle East countries where rates of complete adherence to practice guidelines were 0 [7], and $0.3 \%$ [8] in Jordan and Iran, respectively. In addition, other studies carried out in Western countries reported that adherence to antimicrobial prophylaxis guidelines were relatively higher than those found for Middle Eastern countries [9,10].

Of all the patient files reviewed, $97.8 \%$ received antibiotic prophylaxis before operations. Similar studies in Belgium and Greece reported reported 71 and $70 \%$, respectively[11,12]. In this study only $30.4 \%$ of patients were given prophylactic dose on time as stipulated in the guidelines. This represent a very low percentage compared to other studies which reported $100 \%$ of patients as having received the dose on time [12]. This should attract attention of who should be designated to enforce the routine of initiation of prophylaxis at the time of induction of anesthesia by the anesthesiologist.

Post-operation prophylactic dose was appropriately given to $79.3 \%$ of patients with $15.2 \%$ of the patients switched from appropriate antibiotic to an inappropriate agent. Switches were made from a second generation cephalosporin (cefuroxime) to different combinations This unexplained switch to third-generation cephalosporin or broad spectrum combinations is not recommended for SSI prophylaxis since they have less activity against Staphylococci and may induce the emergence of resistant organisms as well as increase the cost burden [13].

Total duration of antimicrobial prophylaxis was in accordance with guidelines in $67.4 \%$ of patients and but longer in $32.6 \%$ of the. Nonetheless, one or two doses are often enough to achieve antimicrobial prophylaxis over a 48-h period [14]. Most surgeons perceive that there is a need for prolonged administration of antimicrobial prophylaxis [15] and therefore, often use their own judgment. It appears that they have difficulty in accepting the new evidence about antibiotic prophylaxis [16].

In this study, adherence to all the guidelines' recommendations was not completely achieved in any patient enrolled in the study. However, there appears to be better adherence in the dosing of prophylactic agents used and duration of antibiotic use, but lower than figures from other studies that reported 100.0 and $99.1 \%$, respectively) [7].

This seems to indicate minimal awareness on the part of surgeons regarding the importance of giving antimicrobial prophylaxis to prevent SSI in cardiac surgeries compared to other countries.

Assessing the feasibility, appropriateness and acceptance of clinical guidelines among surgeons is very important to ensure effective implementation [17]. We can conclude that if adherence to such guidelines is not improved, the incidence of surgical-site infections may increase and lead probably to increase in antimicrobial resistance [18].

Development and implementation of standard treatment protocols are the best approach to improving surgeons' adherence to guidelines, either by adopting international guidelines or by developing a suitable local one. This should be done with the contribution of surgeons as well as anesthesiologists, microbiologists, pharmacists and infection control departments. These guidelines should take into consideration hospital-specific 
bacterial epidemiological patterns and secure the consensus of all surgeons working in the hospital before its implementation [7].

Clinical pharmacists also need to play an important role in the implementation of such guidelines as they should be charged with the responsibility of ensuring administration of prophylactic dose to patients' prior to and after surgery. As demonstrated by Prado et al in his study which showed that pharmacists made a big improvement when they took charge of the administration of antimicrobial prophylaxis and assessing the appropriateness of the indications; adherence increased from 56 to $100 \%$ while the costs was decreased by $40 \%$ [19].

Although the cost analysis for developing SSI was not addressed in our study, SSI is a cost burden on the healthcare system. It has been reported that surgical-site infections could be costing European healthcare systems between 1.47 and 19.1 billion euros each year [20].

\section{Limitations of the study}

The small number of patients involved in the study does not permit drawing inferences that applies to the entire population. A larger study is needed to assess antimicrobial prophylaxis practice in several hospitals and to be able to generalize our findings to the whole country. As this study is a retrospective study, we assume that our data is accurate, as recorded by anesthesiologists and surgeons in patients' charts.

\section{CONCLUSION}

Adherence to the international antimicrobial prophylaxis guidelines for cardiac surgery was found to be suboptimal in the investigated hospital in Dubai. The results highlight the challenges faced in transferring evidence-based protocol to clinical practice. Various interventions are needed to overcome this problem. Developing local evidence-based protocols in collaboration with surgeons, and strengthening regulations would be important in ensuring adherence to these guidelines. Conducting several seminars to discuss and analyze antibiotic prescribing patterns and increase outcomebased research, as well as documenting benefits and cost effectiveness of appropriate antibiotic use are also important in improving antibiotic prescribing in hospital settings. In addition, the level of adherence to antibiotic use in cardiac surgery guidelines could be optimized by integrating clinical pharmacists into the practice by playing a role in the administration, monitoring, and intervention of antimicrobial prophylaxis.

\section{ACKNOWLEDGEMENT}

The authors would like to thank Prof Saeed Ahmed Khan - Dean of Dubai Pharmacy College - for facilitating this project. Special thanks also go to the following undergraduate students who variously contributed to this work: Lama Adnan, Maymona Abdel Rahman and Huda Mohammed.

\section{REFERENCES}

1. Mangram AJ, Horan TC, Pearson ML, Silver LC, Jarvis WR. Guideline for prevention of surgical site infection, 1999. Hospital Infection Control Practices Advisory Committee. Infect Control Hosp Epidemiol 1999; 20: 250-278.

2. Tjang $Y S$, van $H Y$, Korfer $R$, Grobbee $D E$, van der Heijden GJ. Predictors of mortality after aortic valve replacement. Eur J Cardiothorac Surg 2007; 32: 469-474.

3. Edwards FH, Engelman RM, Houck P, Shahian DM, Bridges CR. The Society of Thoracic Surgeons Practice Guideline Series: Antibiotic Prophylaxis in Cardiac Surgery, Part I: Duration. Ann Thorac Surg 2006; 81: 397-404.

4. Engelman R, Shahian D, Shemin R, Guy TS, Bratzler $D$, Edwards $F$, Jacobs $M$, Fernando $H$, Bridges C. The Society of Thoracic Surgeons practice guideline series: Antibiotic prophylaxis in cardiac surgery, part II: Antibiotic choice. Ann Thorac Surg 2007; 83: 1569-1576.

5. Eagle KA, Guyton RA, Davidoff R, Edwards FH, Ewy GA, Gardner TJ, Hart JC, Herrmann HC, Hillis LD, Hutter AM, Jr.et al. ACC/AHA 2004 guideline update for coronary artery bypass graft surgery: a report of the American College of Cardiology/American Heart Association Task Force on Practice Guidelines (Committee 
to Update the 1999 Guidelines for Coronary Artery Bypass Graft Surgery). Circulation 2004; 110: e340-e437.

6. Bratzler DW, Houck PM. Antimicrobial prophylaxis for surgery: an advisory statement from the National Surgical Infection Prevention Project. Am J Surg 2005; 189: 395-404.

7. Al-Momany NH, Al-Bakri AG, Makahleh ZM, Wazaify MM. Adherence to international antimicrobial prophylaxis guidelines in cardiac surgery: a Jordanian study demonstrates need for quality improvement. J Manag Care Pharm 2009; 15 : 262-271.

8. Askarian M, Moravveji AR, Mirkhani H, Namazi S, Weed $H$. Adherence to American Society of Health-System Pharmacists surgical antibiotic prophylaxis guidelines in Iran. Infect Control Hosp Epidemiol 2006; 27: 876-878.

9. Voit SB, Todd JK, Nelson B, Nyquist AC. Electronic surveillance system for monitoring surgical antimicrobial prophylaxis. Pediatrics 2005; 116: 1317-1322.

10. van Kasteren ME, Kullberg BJ, de Boer AS, Mintjesde GJ, Gyssens IC. Adherence to local hospital guidelines for surgical antimicrobial prophylaxis: a multicentre audit in Dutch hospitals. J Antimicrob Chemother 2003; 51: 1389-1396.

11. Kurz $X$, Mertens $R$, Ronveaux O. Antimicrobial prophylaxis in surgery in Belgian hospitals: room for improvement. Eur J Surg 1996; 162: 15-21.

12. Tourmousoglou CE, Yiannakopoulou EC Kalapothaki V, Bramis J, St PJ. Adherence to guidelines for antibiotic prophylaxis in general surgery: a critical appraisal. J Antimicrob Chemother 2008; 61: 214-218.

13. Martin C, Pourriat JL. Quality of perioperative antibiotic administration by French anaesthetists. J Hosp Infect 1998; 40: 47-53.
14. Bratzler DW, Houck PM, Richards C, Steele L, Dellinger EP, Fry DE, Wright $C$, Ma A, Carr K, Red L. Use of antimicrobial prophylaxis for major surgery: baseline results from the National Surgical Infection Prevention Project. Arch Surg 2005; 140: 174-182.

15. Burke JP. Infection control - a problem for patient safety. N Engl J Med 2003; 348: 651-656.

16. "Timing is everything: Delivering drug prophylaxis to prevent SSls. (surgical-site infections)." Hospital Infection Control and Prevention. AHC Media L.L.C. 2005. Retrieved November 21, 2011 from HighBeam Research. Available from: http://www.highbeam.com/doc/1G1134048651.html

17. Davis DA, Taylor-Vaisey A. Translating guidelines into practice. A systematic review of theoretic concepts, practical experience and research evidence in the adoption of clinical practice guidelines. CMAJ 1997; 157: 408-416.

18. Terpstra S, Noordhoek GT, Voesten HG, Hendriks B, Degener JE. Rapid emergence of resistant coagulase-negative staphylococci on the skin after antibiotic prophylaxis. J Hosp Infect 1999; 43: 195-202.

19. Prado MA, Lima MP, Gomes IR, Bergsten-Mendes $G$. The implementation of a surgical antibiotic prophylaxis program: the pivotal contribution of the hospital pharmacy. Am J Infect Control 2002; 30: 49-56.

20. Leaper DJ, van GH, Reilly J, Petrosillo N, Geiss HK, Torres AJ, Berger A. Surgical site infection - a European perspective of incidence and economic burden. Int Wound J 2004; 1: 247273. 